NOTA CIENTÍFICA

\title{
TRANSIÇÃO DAS FASES FENOLÓGICAS DE NECTANDRA LANCEOLATA NESS E NECTANDRA MEGAPOTAMICA (SPRENG.) MEZ (LAURACEAE) EM DIFERENTES ÁREAS NO MUNICÍPIO DE JOAÇABA
}

\author{
Bagatini KP*, Olivo E* ${ }^{* *}$ Oliveira LM**
}

Resumo

Objetivou-se acompanhar aspectos da fenologia de Nectandra megapotamica e Nectandra lanceolata. A observação ocorreu em três áreas em Joaçaba, SC, no período de maio de 2013 a fevereiro de 2014, com periodicidade mensal em estádio vegetativo e quinzenal em reprodutivo. A floração de N. megapotamica ocorreu de agosto a outubro, e a maturação dos frutos, até fevereiro. O índice de Fournier demonstrou baixa produtividade ( $24 \%$ de antese e $19 \%$ de fruto). A floração de N. lanceolata ocorreu de setembro a dezembro, com frutos até fevereiro, também com baixa produtividade ( $14 \%$ de antese e 9\% de fruto). É necessário acompanhamento por um período de tempo maior. Palavras-chave: Floração. Frutificação. Disponibilidade de sementes. Espécies florestais.

\footnotetext{
* Mestre em Botânica pela Universidade Federal do Paraná; Graduada em Ciências Biológicas pela Universidade do Oeste de Santa Catarina; Professora da Universidade do Oeste de Santa Catarina de Joaçaba; Rua Getúlio Vargas, 2125, Flor da Serra, 89600-00, Joaçaba, Santa Catarina, Brasil; katiane.bagatini@unoesc.edu.br

${ }^{* *}$ Graduanda em Ciências Biológicas pela Universidade do Oeste de Santa Catarina; ediana.olivo@yahoo.com

${ }^{* * *}$ Pós-doutora e Doutora em Agronomia (Fitotecnia-Sementes) pela Universidade Federal de Lavras; Professora efetiva da Universidade do Estado de Santa Catarina - Centro de Ciências Agro veterinárias; a2lmo@cav.udesc.br
} 


\section{Preliminary assessment of the transition between the phases of phenological nectandra lanceolata ness and nectandra megapotamica (spreng.) mez (lauraceae) in Joaçaba}

\section{Abstract}

The aim of the study was to monitor aspects of phenology of Nectandra megapotamica and Nectandra lanceolata. The observation tookplace in three areas in Joaçaba, SC in theperiod from May, 2013 to February, 2014, monthly in vegetative stage and fortnightly in breeding. The flowering of N. megapotamica occurred from August through October, the fruit ripening until February. The index Fournier demonstrated low productivity (24\% of anthesis and 19\% fruit). The flowering of N. lanceolata occurred from September to December, with fruit until February, also with low productivity (14\% anthesis and 9\% fruit). Monitoring for a longer period of time is required.

Keywords: Flowering. Fruiting. Availability of seeds. Forest species.

\section{INTRODUÇÃO}

Fenologia é o estudo da ocorrência de eventos biológicos sazonais relacionados às mudanças nos meios abiótico e biótico, que busca compreender os ciclos de vida. ${ }^{1}$ Entre esses eventos, o período reprodutivo da espécie, na população ou em populações distintas, destaca-se como um dos principais eventos, dessa forma, há a necessidade de conhecimento, uma vez que é este que vai condicionar a disponibilidade de recursos para a manutenção das áreas florestais. ${ }^{2,3}$ Assim, o detalhamento de aspectos fenológicos regionais é importante, uma vez que as espécies podem apresentar diferenças cronológicas e de periodicidade de acordo com o ecossistema em que ocorrem. ${ }^{4}$

Para o Município de Joaçaba, até o presente momento, não há relatos de estudos fenológicos realizados em espécies florestais e, em nível nacional, aspectos sobre a fenologia de espécies florestais ainda são escassos, tendo sido realizados para Aniba duckei Kostermans (Lauraceae), ${ }^{5}$ Copaifera langsdorffii Desf. (Fabaceae), ${ }^{4}$ espécies de Rubiaceae, ${ }^{6}$ de Nectandra megapotamica e de N. lanceolata (Lauraceae) ${ }^{7,8}$ As espécies-alvo do presente trabalho, Nectandra lanceolata e N. megapotamica (Lauraceae), vêm sendo monitoradas, também, em relação a aspectos fenológicos desde 2007 no Município de Colombo, PR. ${ }^{7,8}$ No entanto, até o momento, os dados publicados são escassos.

A necessidade de conhecimento e o desenvolvimento de metodologias eficazes na obtenção de sementes e na produção de mudas de espécies florestais nativas justificam o presente trabalho. Dessa forma, este estudo teve por objetivo abordar aspectos preliminares sobre a transição das fases fenológicas vegetativa e reprodutiva das espécies Nectandra megapotamica (Spreng.) Mez e Nectandra lanceolata Nees (Lauraceae). 


\section{MATERIAL E MÉTODOS}

Foram amostradas três áreas para a coleta dos dados no Município de Joaçaba, SC (Tabela 1). As observações foram coletadas no período que corresponde a maio de 2013 a fevereiro de 2014.

Tabela 1 - Número de indivíduos demarcados de Nectandra megapotamica (Spreng.) Mez e Nectandra lanceolata Nees (Lauraceae) nas áreas de estudos com as respectivas coordenadas geográficas

\begin{tabular}{cccc}
\hline & N. megapotamica & N. lanceolata & Coordenadas geográficas \\
\hline Área 1 & 9 & 18 & 22JDQ 41658 MGRS 95710 \\
Área 2 & 16 & 5 & 22JDQ 48255 MGRS 95748 \\
Área 3 & 7 & 7 & 22JDQ 48960 MGRS 94638 \\
\hline
\end{tabular}

N. total de indivíduos

$32 \quad 30$

Fonte: os autores.

A partir de observações in loco, pode-se constatar que a área 1 se caracteriza por ser uma unidade de conservação, com mata densa; a área 2 é muito fragmentada, muitos indivíduos estão situados em locais de alta declividade e a maioria em condição semiaberta ou aberta; e a área 3 é uma mata ciliar urbana. O clima das áreas classifica-se como subtropical úmido, segundo classificação de Köppen-Geiger. ${ }^{9}$ Os dados meteorológicos foram obtidos por meio do Instituto Nacional de Meteorologia (Figura 1). ${ }^{10}$

Figura 1 - Referente ao período de maio de 2013 a fevereiro de 2014

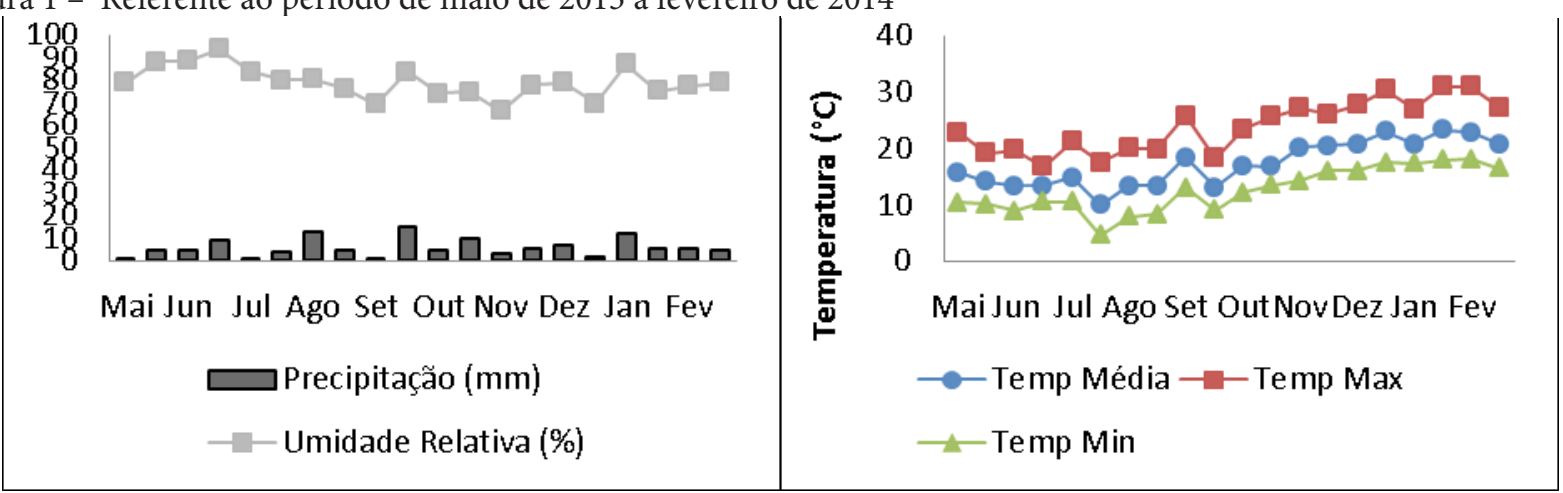

Fonte: Instituto Nacional de Meteorologia. ${ }^{10}$

Todos os indivíduos selecionados apresentavam circunferência à altura do peito (CAP) mínima de $15 \mathrm{~cm}$. Na fase vegetativa, as caracterizações dos indivíduos foram mensais e, após o surgimento das primeiras flores, a frequência de visitações passou a ser quinzenal. Para a caracterização foram consideradas as seguintes fenofases: fase vegetativa; botão floral; antese; frutos imaturos; frutos imaturos crescidos; frutos maduros; frutos caídos no chão; desfolhamento e enfolhamento. Para quantificar as fenofases foi utilizada a metodologia proposta por Fournier, ${ }^{11}$ obedecendo a uma escala de 0 a 4 , sendo 0 - ausência da fenofase; 1 - presença da fenofase com magnitude entre 1 e 25\%; 2 - presença da fenofase entre 26 e 50\%; 3 - presença da fenofase entre 51 e 75\% e 4 - presença da fenofase entre 76 e $100 \%$. 
Considerando a intensidade de cada fenofase, foi calculada a porcentagem de Fournier por meio da seguinte fórmula: $\left[\%\right.$ de Fournier $=\left(\sum\right.$ Fournier $\left.\left./ 4 \mathrm{~N}\right) \times 100\right] .{ }^{11}$ A normalidade da distribuição dos dados foi testada utilizando-se o teste de Shapiro-Wilk. Como os dados têm distribuição normal, foi utilizada a análise de Correlação de Spearman (rs) entre o número de indivíduos e a porcentagem de Fournier de cada fenofase e as variáveis climáticas. As análises estatísticas foram realizadas por intermédio do programa BioStatic 5.0 $0^{\circledR}$

\section{RESULTADOS E DISCUSSÃO}

\subsection{NECTANDRA MEGAPOTAMICA}

A floração da N. megapotamica teve início no mês de agosto e término no mês de outubro (Figuras 2A e 2B). O período de floração observado no presente estudo é similar ao descrito por Souza e Moscheta ${ }^{12}$ e Rego ${ }^{7}$ para a espécie, nos Municípios de Maringá e Colombo, PR, respectivamente. Ao comparar os resultados obtidos no presente estudo com o trabalho de Souza e Moscheta, ${ }^{12}$ observa-se que o período que corresponde ao início da floração foi o mesmo, no entanto, o tempo de floração variou, visto que as plantas de N. megapotamica caracterizadas em Joaçaba apresentaram maior intervalo entre o início e o fim da floração. Já Rego ${ }^{7}$ verificou flores em N. megapotamica de junho a setembro, ou seja, dois meses de antecedência quando comparados aos dados do presente trabalho.

Figura 2 - Aspectos fenológicos de Nectandra megapotamica (Spreng.) Mez (Lauraceae) nas áreas 1 (pontos redondos), 2 (pontos quadrados) e 3 (pontos triangulares). (A) Intensidade de botões florais, indicada pelo índice de Fournier, (B) Intensidade de antese, indicada pelo índice de Fournier, (C) Intensidade de frutos, indicada pelo índice de Fournier e (D) Porcentagem de indivíduos floridos
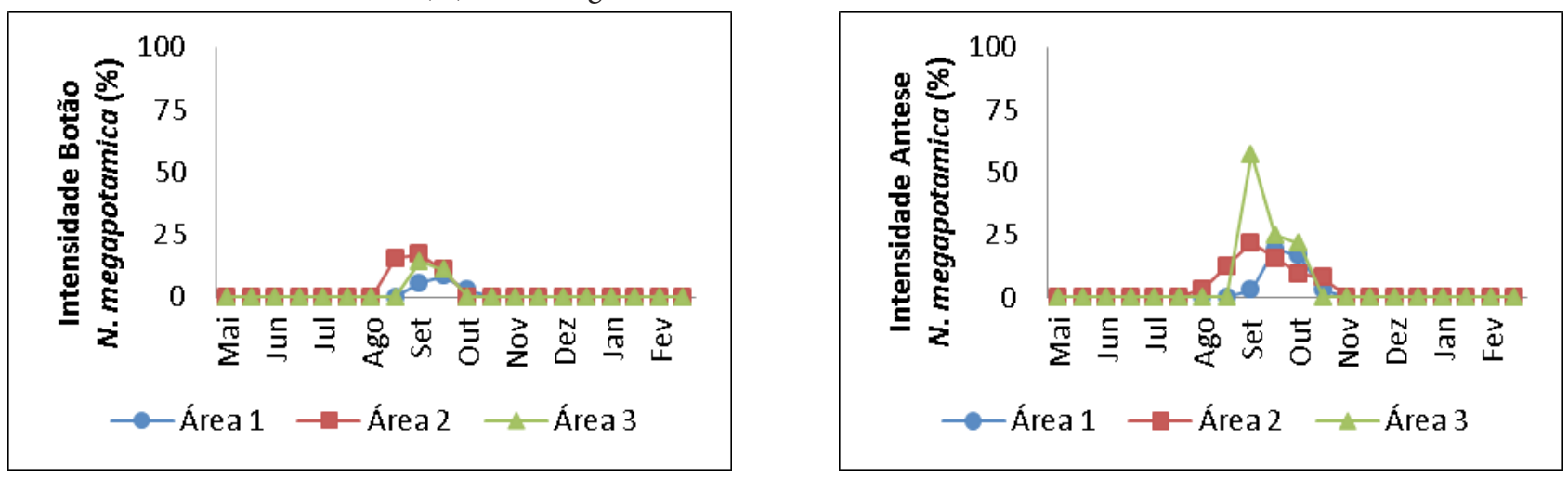


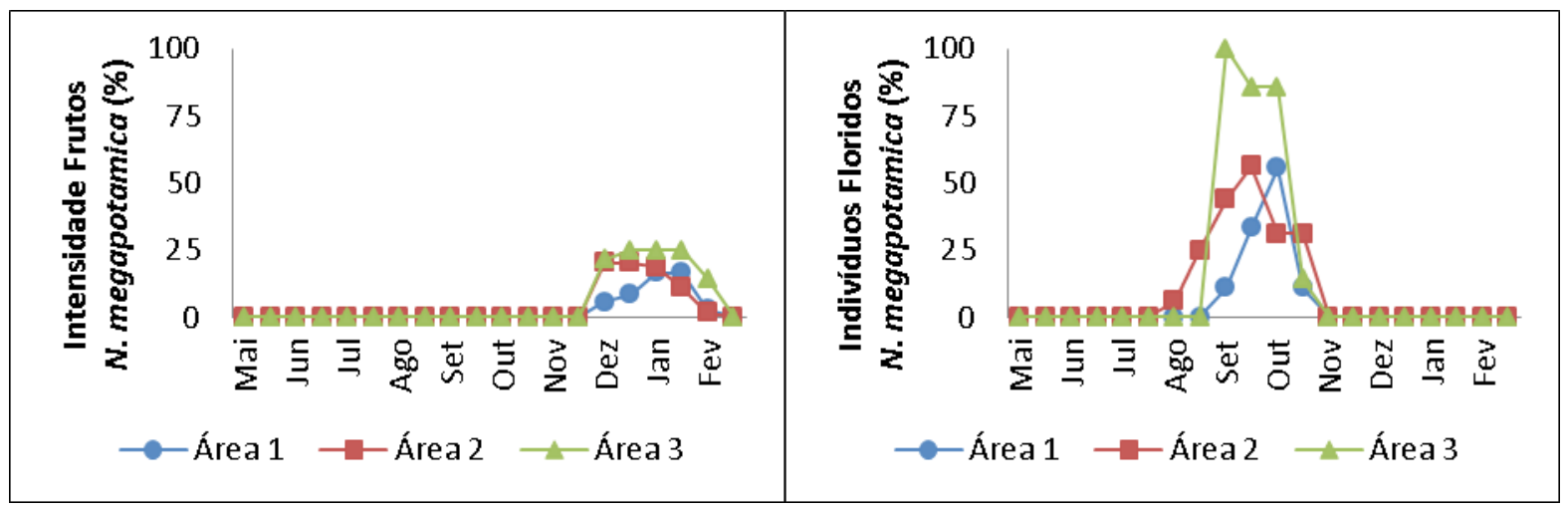

Fonte: os autores.

A partir de outubro, iniciou-se o desenvolvimento dos frutos, os quais se apresentaram completamente desenvolvidos, com início da maturação no mês de dezembro, e mantiveram-se até fevereiro (Figura 2C). De acordo com Rego, ${ }^{7}$ a frutificação acontece de outubro a fevereiro. No presente estudo, a frutificação ocorreu no período de temperatura mais elevada, e a maturação dos frutos coincidiu com o aumento da temperatura (Figura 1).

Por meio do teste de Correlação de Spearman (rs), entre as variáveis porcentagem de Fournier em cada fenofase e as variáveis climáticas, foi constatada forte correlação positiva entre folhas novas ( $r s=0.9813 ; \mathrm{p}=0.0001$ ), frutos crescidos ( $r s=0.7184 ; \mathrm{p}=0.0017)$, frutos maduros ( $r s=0.7830 ; \mathrm{p}=0.0003$ ) e frutos caídos ( $r=0.7830 ; \mathrm{p}=0.0003$ ) e a temperatura, e fraca correlação positiva entre a antese e a precipitação $(\mathrm{rs}=0.4993 ; \mathrm{p}=0.0489)$. Isso pode estar relacionado à sazonalidade apresentada pela espécie, a qual, por sua vez, é o resultado da evolução destas em resposta às condições físico-químicas e biológicas do local. ${ }^{1}$

A visualização de frutos maduros ainda nas árvores foi muito baixa, na maioria das vezes foram observados já no chão, o que indica que a permanência dos frutos maduros ocorre durante um período de tempo reduzido. Outra explicação para a pequena quantidade de frutos nas árvores é o fato de a espécie ser atrativa à avifauna, sendo consumida assim que ocorre a alteração da coloração. ${ }^{7}$ Foi observado que na segunda quinzena de setembro (Figura 1), a precipitação pluvial foi elevada, atingindo $44 \mathrm{~mm}$, momento em que a floração se encontrava no auge, fato que pode ter ocasionado a queda de muitas flores, afetando, assim, a produção de frutos. A queda de estruturas reprodutivas pode estar associada às condições climáticas, como ocorre na região Amazônica. ${ }^{13}$

Em relação aos indivíduos floridos, a área 3 apresentou maior quantidade total (100\%) quando comparada às áreas 1 (55\%) e 2 (69\%); o pico de floração na área 1 ocorreu em outubro (55\%), e nas áreas 2 e 3, ocorreu em setembro (56 e 100\%) (Figura 2D). Entre as áreas, a maior ocorrência de floração na área 3 pode estar relacionada ao fato de todos os indivíduos estarem situados em borda de mata, recebendo grande intensidade de luz solar. 


\subsection{NECTANDRA LANCEOLATA}

A floração teve início no final do mês de setembro e término no mês de dezembro (Figuras 3A e 3B). O desenvolvimento dos frutos ocorreu de novembro a fevereiro, quando foram observados maduros e caídos no chão (Figuras 3C e 3D). A frutificação procedeu-se no período de temperatura mais elevada, média de $22,09^{\circ} \mathrm{C}$ em janeiro (Figura 1). Os dados obtidos no presente trabalho são iguais aos observados por Rego, ${ }^{8}$ no Município de Colombo, PR, o qual identificou que a espécie floresce de setembro a dezembro e frutifica de janeiro a março, e diferem do observado por Carvalho, ${ }^{14}$ que cita a floração de dezembro a janeiro para o Estado de Santa Catarina.

Por meio do teste de Correlação de Spearman, pode-se constatar uma forte correlação positiva entre folhas novas ( $\mathrm{rs}=0.9732 ; \mathrm{p}=0.0001)$, folhas velhas $(\mathrm{rs}=-0.9836 ; \mathrm{p}=0.0001)$ e fruto crescido $(\mathrm{rs}=0.7911 ; \mathrm{p}=0.0003)$ e a temperatura, enquanto as fenofases de frutos maduros $(\mathrm{rs}=0.5807 ; \mathrm{p}=0.0183$ ) e caídos ( $\mathrm{rs}=0.5807 ; \mathrm{p}=0.0183$ ) têm correlação mediana positiva com a temperatura. Tanto em $N$. megapotamica quanto em $N$. lanceolata a temperatura está relacionada à formação de folhas novas e ao desenvolvimento dos frutos. Meses com maiores temperaturas apresentam maior percentual de espécies com atividade reprodutiva em Floresta Ombrófila Densa. ${ }^{13,15}$

Figura 3 - Aspectos fenológicos de Nectandra lanceolata Ness (Lauraceae) nas área 1 (pontos redondos), 2 (pontos quadrados) e 3 (pontos triangulares). (A) Intensidade de botões florais, indicada pelo índice de Fournier, (B) Intensidade de antese, indicada pelo índice de Fournier, (C) Intensidade de frutos, indicada pelo índice de Fournier, (D) Porcentagem de indivíduos com frutificação e (E) Porcentagem de indivíduos floridos

\begin{tabular}{|c|c|c|}
\hline 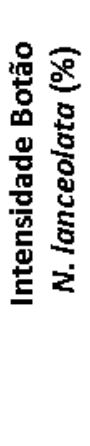 & 25 & 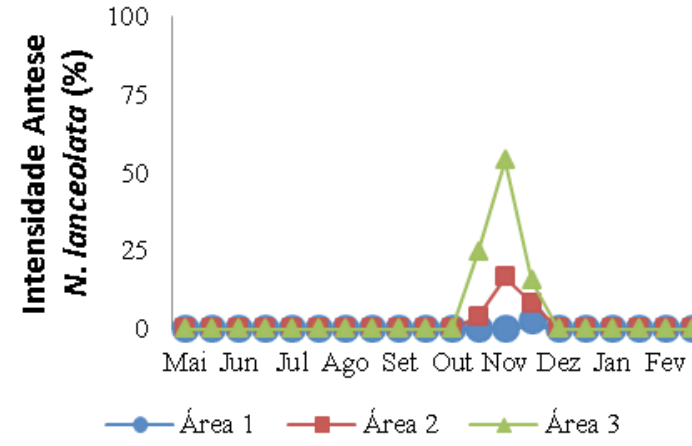 \\
\hline 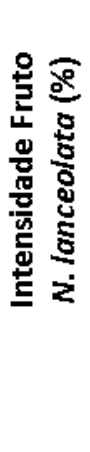 & 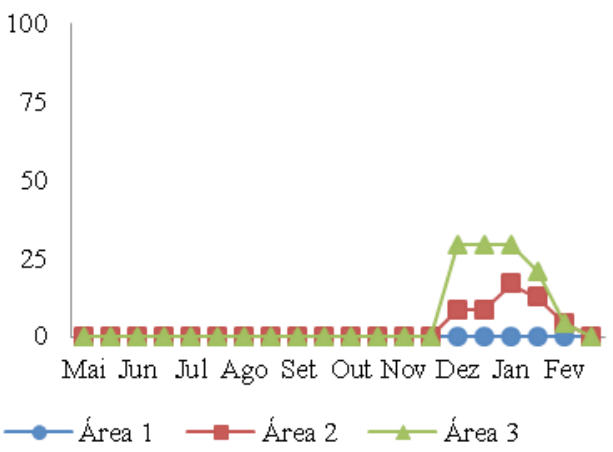 & 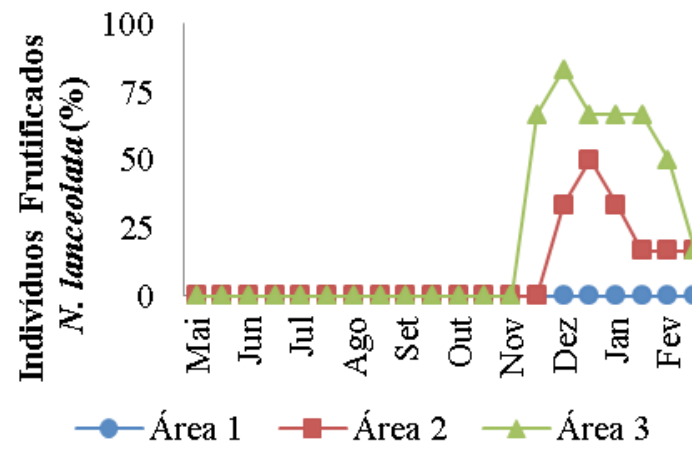 \\
\hline
\end{tabular}




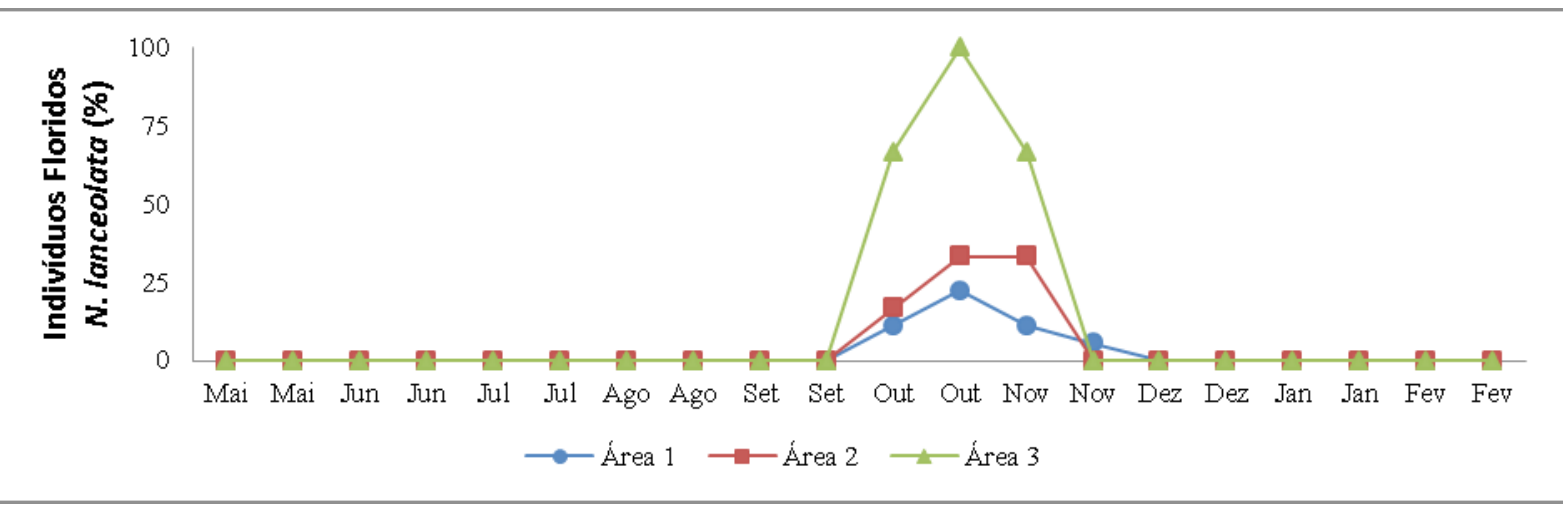

Fonte: os autores.

Na área 1 ocorreu a formação de flores, no entanto, não foi registrada a presença de frutos, possivelmente, pelo abortamento de frutos ou pela queda destes. Referências que confirmem o abortamento ou a queda de frutos na família Lauraceae são inexistentes. No entanto, a baixa produção de frutos é citada para outra espécie da família Lauraceae (Aniba duckei). ${ }^{5}$ Os autores conseguiram relacionar o baixo índice de floração à não ocorrência de formação de frutos, assim como observaram que plantas com eventos de floração por três anos seguidos não frutificaram.

A área 1 apresentou a menor quantidade total de indivíduos floridos (11\%), seguida da área $2(33 \%)$ e da área $3(100 \%)$, visto que o pico de floração na área 1 ocorreu no mês de novembro (11\%) e nas áreas 2 e 3, ocorreu no mês de outubro (33 e 100\%) (Figura 3E). Pôde-se observar que os indivíduos com frutificação estão localizados na borda, o que pode estar relacionado à grande intensidade de luz solar, quando comparados aos indivíduos que estão dentro da mata. A área 3 teve maior frutificação tanto para N. lanceolata (Figura 3D) quanto para N. megapotamica (Figura 2C), o que pode estar relacionado à luminosidade da área. Em estudo realizado por Alberti e Morelatto, ${ }^{16}$ constatou-se que os frutos de N. megapotamica ocorrem com tamanho e em quantidades diferentes dependendo do local onde se encontram; no presente estudo, observou-se que em ambientes de maior luminosidade ocorreu maior produção de frutos em comparação com indivíduos de outras áreas.

A ocorrência de eventos reprodutivos nos indivíduos de N. lanceolata foi relativamente alta quando comparada ao observado para o gênero Nectandra (5,5\%) em estudo proposto por Mantovani et al., ${ }^{15}$ em Floresta Ombrófila Densa. Espécies que apresentam baixa quantidade de indivíduos com eventos reprodutivos anuais têm maior necessidade de manutenção das matrizes em planos de manejo para a obtenção de sementes e a diminuição dos efeitos da endogamia na população. ${ }^{15}$

\section{CONSIDERAÇÕES FINAIS}

O local de ocorrência afetou as fases fenológicas; foi observada maior quantidade de indivíduos na condição borda em processo de floração e frutificação. Tanto N. megapotamica quanto N. lanceolata tiveram uma diminuição da quantidade de estruturas reprodutivas quando da passagem entre as 
fenofases, ou seja, a produção de flores foi maior do que a de frutos, e a quantidade de frutos imaturos foi maior do que a de maduros. Em ambas as espécies, o fator temperatura atuou na produção e maturação dos frutos. O acompanhamento durante o período de 10 meses foi insuficiente para a compreensão de eventos supra-anuais, bem como para a comprovação dos fatores envolvidos nos eventos anuais.

\section{REFERÊNCIAS}

1. Odum EP. Fundamentos de ecologia. 7a. ed. Lisboa: Fundação Calouste Gulbenkian; 2004.

2. Eça-Neves FF, Morellato LPC. Métodos de amostragem e avaliação utilizados em estudos fenológicos de florestas tropicais. Acta Botânica Brasílica. 2004; 8(1):99-108.

3. Cesário LF, Gaglianone MC. Biologia floral e fenologia reprodutiva de Schinus terebinthifolius Raddi (Anacardiaceae) em Restinga do Norte Fluminense. Acta Botânica Brasílican. 2008; 22(3):828-833.

4. Pedroni F, Sanchez M, Santos FAM. Fenologia da copaíba (Copaifera langsdorffii Desf. Leguminosae, Caesalpinioideae) em uma floresta semidecídua no sudeste do Brasil. Revista Brasileira de Botânica. 2002; 25(2):183-194.

5. Magalhães LMS, Alencar JC. Fenologia de pau-rosa (Aniba duckei Kostermans), Lauraceae, em floresta primária da Amazônia Central. Acta Amazonica. 1979; 9(2):227-232.

6. Martin-Gajardo IS, Morellato LPC. Fenologia de Rubiaceae do sub-bosque em floresta Atlântica no sudeste do Brasil. Revista Brasileira de Botânica. 2003; 26(3):299-309.

7. Rego GM. Monitoramento da fenologia de espécies arbóreas das florestas Brasileiras: Canelaimbuia. Colombo: Embrapa Florestas; 2009.

8. Rego GM. Monitoramento da fenologia de espécies arbóreas das florestas Brasileiras: Canelabranca. Colombo: Embrapa Florestas; 2010.

9. Pandolfo C, Braga HJ, Silva Junior VP, Massignam AM, Pereira ES, Thomé VMR, et al. Atlas climatológico digital do Estado de Santa Catarina. Florianópolis: Epagri; 2002.

10. Instituto Nacional de Metereologia. BDMEP - Banco de Dados Meteorológicos para Ensino e Pesquisa. [acesso em 2013 nov 01]. Disponível em: www.inmet.gov.br/portal/index.php?r=bdmep

11. Fournier LA. Un metodo cuantitativo para la medición de características fenológicas en arboles. Turrialba. 1974; 24(4):422-424.

12. Souza LA, Moscheta IS. Morfo-anatomia e aspectos da biologia floral de Nectandra megapotamica (Spreng.) Mez (Lauraceae). Acta bot. Bras. 2000; 14(1):15-25. 
13. Carvalho JOP. Fenologia de espécies florestais de potencial econômico que ocorrem na Floresta Nacional do Tapajós. Belém: Embrapa; 1980.

14. Carvalho PE. Circular Técnica 63: Taxonomia. Colombo: Embrapa Florestas; 2002.

15. Mantovani M, Ruschel AR, Reis MS, Puchalski A, Nodari RO. Fenologia reprodutiva de espécies arbóreas em uma formação secundária de Floresta Atlântica. Sociedade de Investigações Florestais. 2003; 27(4):451-458.

16. Alberti LF, Morelatto LPC. Produção de frutos em indivíduos de Nectandra megapotamica (Sprengel) Mez (Lauraceae) sob diferentes condições ambientais. In: Anais do $7^{\circ}$ Congresso de Ecologia do Brasil, 2005; Caxambu. Minas Gerais; 2005.

Recebido em 11 de abril de 2014 Aceito em 04 de maio de 2015 
